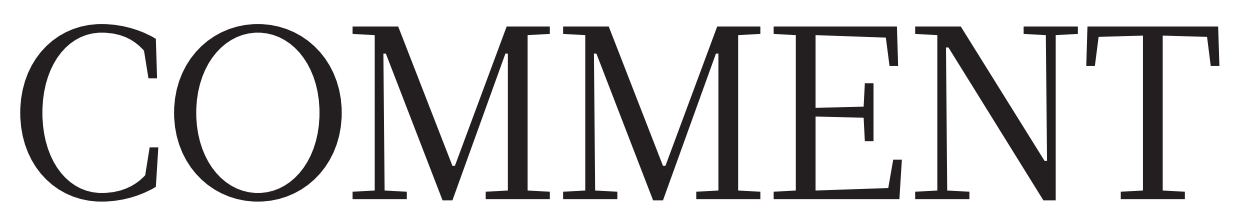

\begin{tabular}{l|l|l|l}
\hline $\begin{array}{l}\text { EXPLORATION Seafarers' } \\
\text { journals are a rich record } \\
\text { of discoveries } \mathbf{p . 3 4 0}\end{array}$ & $\begin{array}{l}\text { OUTBREAKs WHO drafts code } \\
\text { on pathogen sequence } \\
\text { misleading meanings in } \\
\text { sharing } \mathbf{p . 3 4 5}\end{array}$ & $\begin{array}{l}\text { OBITUARY Aaron Klug, } \\
\text { electron-tomography Nobel } \\
\text { laureate, remembered } \mathbf{p . 3 4 6}\end{array}$ \\
\hline
\end{tabular}

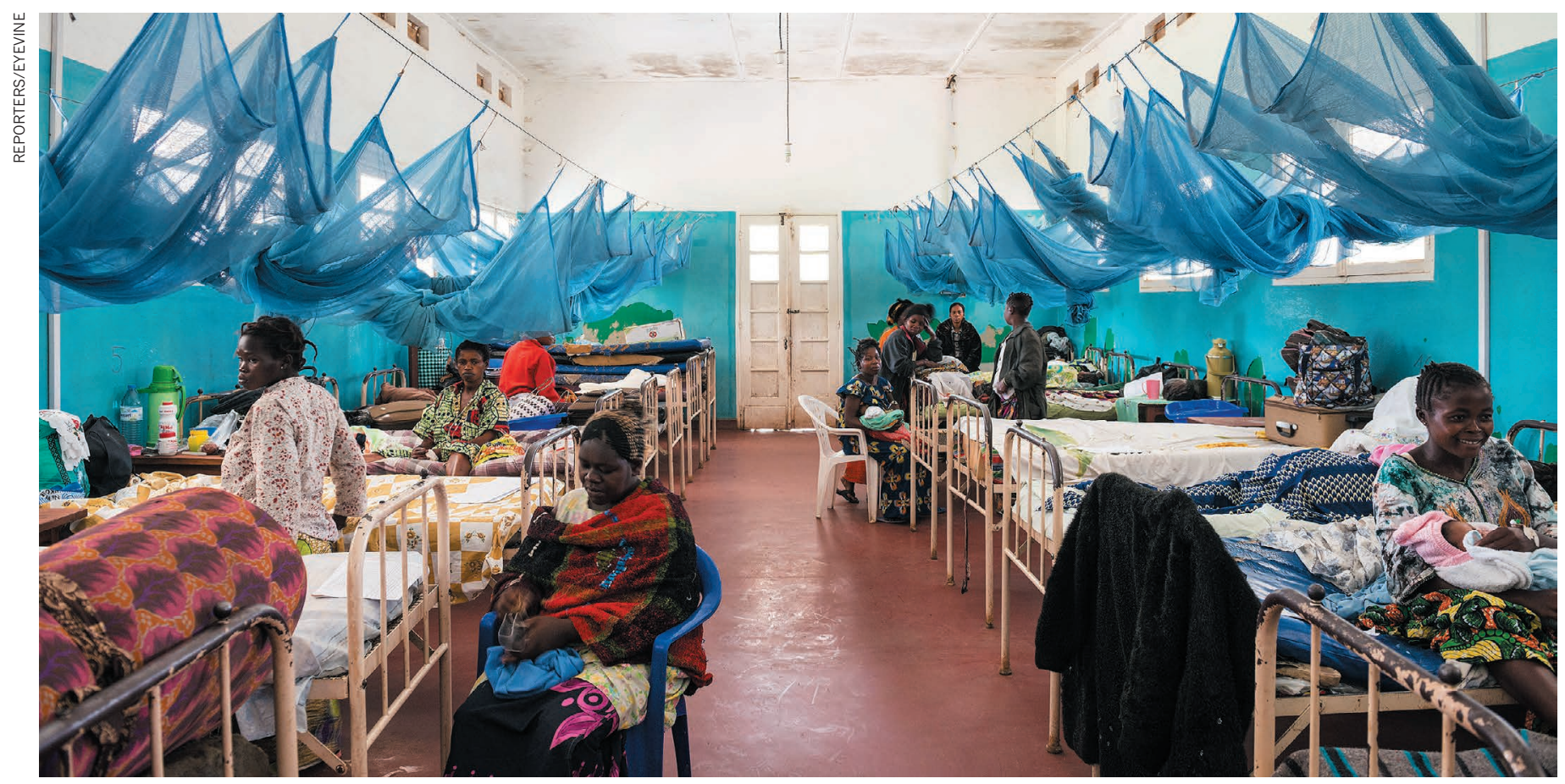

Women in a hospital ward with malaria bed nets in Bunia, the Democratic Republic of the Congo.

\title{
Vaccine candidates for poor nations are going to waste
}

Promising immunizations for diseases that affect mostly people in low- and middleincome countries need help getting to market, urge David C. Kaslow and colleagues.

$\mathrm{S}$ ome 240 vaccine candidates are currently in the development pipeline for diseases such as malaria, tuberculosis and pneumonia - conditions that predominantly affect people in low- and middle-income countries (LMICs). Just two that made it through the pipeline in recent years are widely used in these nations: a conjugate vaccine for meningitis serogroup A diseases (see 'Game changers') and a vaccine against Japanese encephalitis virus.

Drug developers must clear many barriers to get a vaccine licensed so it can be given to millions of people - whether in routine immunizations or during a disease outbreak. The initial phases of development entail evaluating candidates identified from basic research in proof-of-principle clinical trials, usually involving tens or hundreds of people (often called 'the valley of death' because so many candidates fail at this stage owing to a lack of resources; see Nature 453, 840-842; 2008). Next, developers must invest in a manufacturing facility and test candidates in trials involving several thousand to tens of thousands of people (phase III). If regulators approve the vaccine for sale, vaccine manufacturers must then monitor safety in populations that have been vaccinated (known as post-marketing evaluation or phase IV studies).

Over the past decade, billions of dollars have been ploughed into academic laboratories, biotechnology firms and pharmaceutical companies to help them through the first phase of vaccine development for diseases that mainly affect emerging economies. The money has come from many organizations, including the US National Institutes of Health (NIH), the European Union, the Wellcome Trust in London and the Bill \& Melinda Gates Foundation in Seattle, Washington. Numerous candidates are now in proof-of-principle trials, largely thanks to these investments and to advances in technology that span genomics to immunology. Several candidates are ready for late-stage clinical trials. 


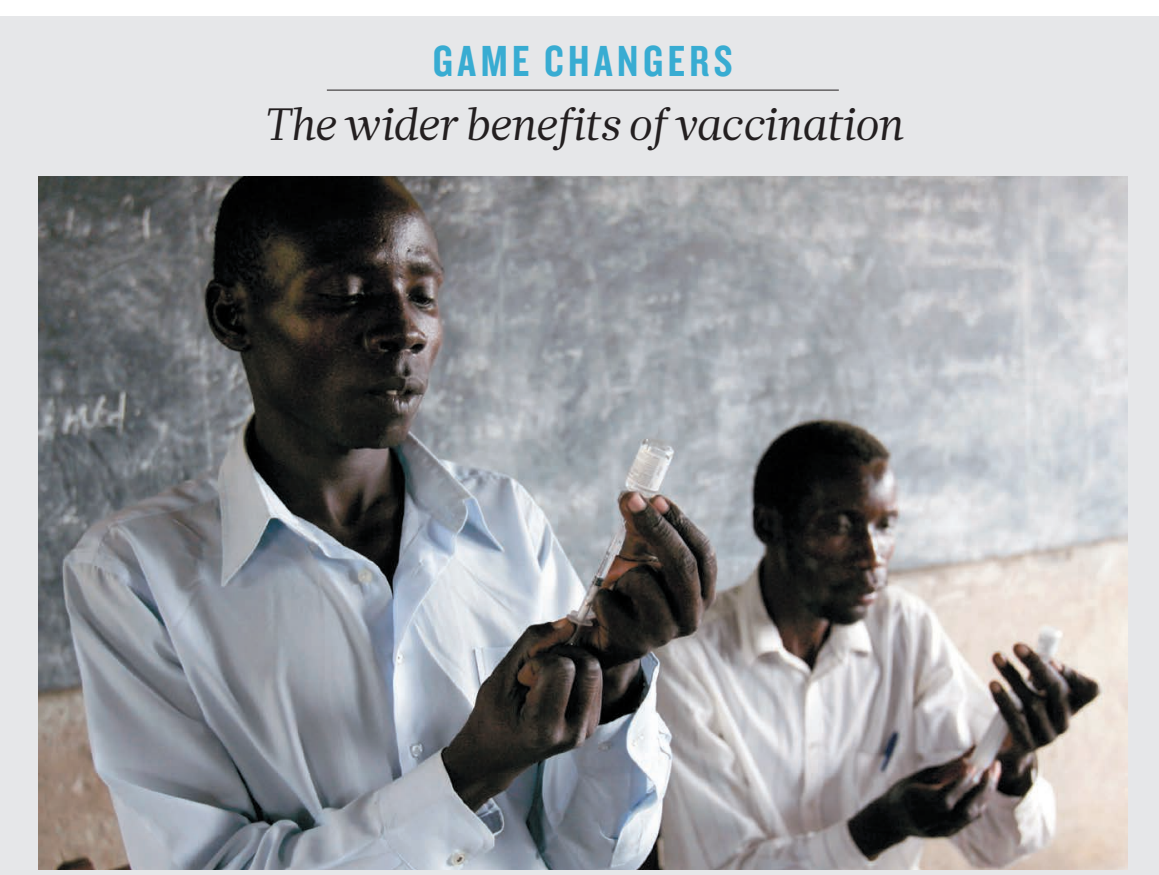

Health workers in Uganda preparing for a meningitis vaccination campaign.

Vaccines are one of the most effective means of improving public health, technically and economically.

They benefit societies as well as individuals. They disrupt pathogen transmission, promote school attendance and attainment, and enable greater labour participation and productivity. In some instances, they have limited the spread of antimicrobial resistance, or even (in the case of smallpox) eradicated a disease ${ }^{7}$.

Between 2010 and 2017, the vaccination of more than 300 million people in sub-Saharan Africa with meningococcal serogroup $A$ conjugate vaccine has almost eliminated meningitis $A^{8,9}$. This disease type was causing hundreds of thousands to fall sick and, during the worst epidemic in 1996-97, resulted in more than 25,000 deaths. It has also removed a major cause of poverty in the region. Meningitis $A$ diseases took up $50 \%$ of families' annual incomes, starting a spiral of poverty from which it was difficult to recover ${ }^{10}$. D.C.K. etal. ret much of this promising pipeline could go to waste. No single organization or group is striving to support the formidably challenging second phase of vaccine development for diseases that mainly affect emerging economies.

\section{LONG ROAD}

Taking a vaccine candidate from a discovery at the lab bench to widespread deployment is complex, lengthy and expensive.

Take the RTS,S vaccine for malaria. In 1967, investigators showed that mice could be protected against rodent malaria by injecting them with a partially inactivated form of the malaria parasite Plasmodium ${ }^{1}$. In 1983, researchers identified the gene encoding a protein on the surface of Plasmodium ${ }^{2}$. This protein is recognized by antibodies that protect rhesus macaques from Plasmodium infection ${ }^{3}$. More than a decade later, studies revealed that a recombinant form of this protein could protect six out of every seven adult human volunteers from infection $^{4}$. Then, in 2004, these findings were confirmed in a study involving more than 2,000 children from Mozambique ${ }^{5}$. From its formal start in the mid-1980s, this first phase of development alone took about 20 years and cost around US\$200 million.

Beginning in 2009, phase III clinical studies were conducted in children from seven countries in sub-Saharan Africa ${ }^{6}$. The trials were run through a collaboration between the drug manufacturer GlaxoSmithKline (where R.R. is chief scientist) and the PATH Malaria Vaccine Initiative (MVI), headquartered in Seattle, Washington (D.K. was also involved in these studies). MVI received grant funding from the Bill \& Melinda Gates Foundation.

In 2015, the vaccine was approved for market by the European Medicines Agency, on one condition: that the developers conduct post-marketing evaluations. But before a vaccine can be given to millions in LMICs, it must be recommended by the World Health Organization (WHO) and approved through the WHO prequalification process. Unexpectedly for the developers, in October 2015, the WHO's Strategic Advisory Group of Experts on Immunization and the Malaria Policy Advisory Committee called for the vaccine to be given on a pilot basis in just three countries: Ghana, Kenya and Malawi.

The post-marketing studies are scheduled to begin next year. But the follow-up pilot studies are a massive undertaking: the relevant infrastructure must be set up in the selected countries. That includes training enough people to collect data in the field, as well as establishing systems to collect, store and analyse the data.

So far, the RTS,S vaccine programme has cost more than $\$ 700$ million. Several hundred million dollars more will be needed for a manufacturing plant (see 'Long and costly').

\section{MARKET FAILURE}

Who is going to cover the costs for other vaccine candidates in the pipeline?

We are most concerned about those that could have a significant impact on public health in LMICs, but that offer no or limited economic return for developers (see 'Spreading the cost').

Since 2000, the number of large companies developing and manufacturing vaccines has fallen from nine to four. (Manufacturers in emerging economies have tended to focus on producing vaccines that have already been developed in high-income countries.) This means that the burden of developing candidates for LMICs is now spread across fewer firms.

Some pharmaceutical giants, such as GlaxoSmithKline and Sanofi, have contributed for decades to the late-stage development of vaccines targeting diseases that mainly (or solely) affect LMICs. But even these firms are becoming more reluctant to invest as candidates are discovered at a faster rate, and as more of those are for diseases that affect only LMICs. As disease outbreaks become more frequent, such companies are similarly becoming averse to reallocating resources and disrupting daily operations to respond to public-health emergencies.

Some vaccine candidates in the current pipeline, such as those for Group A streptococcus, Group B streptococcus and tuberculosis, could have public and private markets in middle-income countries such as China. Yet developing them is a high-risk option. Various factors make it difficult for companies to predict the return on their investments. These can include: knowledge gaps about a candidate's mechanism of action, and an incomplete understanding of the impact of a disease - for instance, on the number of people infected, how many deaths it causes and the effect on a country's economy.

Sixty per cent of the vaccine candidates identified for diseases that affect LMICs target HIV, malaria, tuberculosis and pneumonia - diseases that are a much bigger problem in LMICs than in high-income countries. And nearly $90 \%$ of these candidates are in phase II clinical trials (involving hundreds or a few thousand people) or in 
earlier stages of development.

These candidates could transform public health, but they are not a compelling business case for developers. As long as no organization plans and supports the development of this class of vaccine from beginning to end, it could be many decades before they improve global health and wellbeing. They could even languish altogether.

\section{A WAY FORWARD}

So what should be done? In our view, the main stakeholders must come together to define a new path for the sustainable development of vaccines that are socially justified but that have no business case, an uncertain one, or that require considerable public funding to reach the clinic.

Participants should include funding agencies such as the Wellcome Trust, the Bill \& Melinda Gates Foundation and the NIH; early-development partners such as PATH and the International Vaccine Institute in Seoul; vaccine manufacturers from wealthy and emerging economies; and organizations that recommend and procure vaccines for poor countries, such as the WHO and Gavi, the Vaccine Alliance.

The first aim should be to assess all the candidates in the pipeline. To ensure the best use of limited resources, the focus must be the most favourable candidates that face uncertain business cases. Those that are unlikely to have a significant impact socially and economically must be deprioritized.

Ultimately, the stakeholders must specify which organization, or alliance, should drive development for the prioritized vaccines from beginning to end, and what role each partner should have. They must also lay out the practical steps that are most likely to lead to the successful late-stage development and use of these vaccines - including schemes for resourcing.

\section{LONG AND COSTLY}

The drug giant GlaxoSmithKline (GSK) and others have spent nearly US\$1 billion on developing the RTS,S malaria vaccine. Millions more must be invested before it can be implemented at scale.

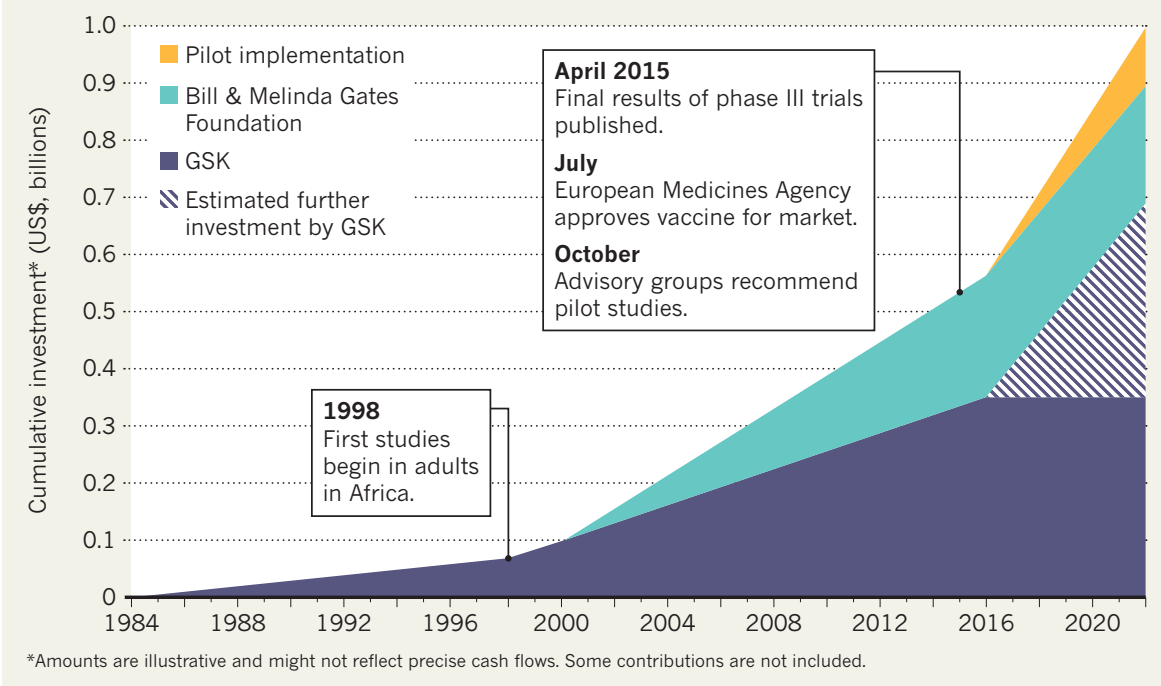

Money is the main limiting factor. In principle, subsidies from governments, such as those of the G20 countries, and philanthropic organizations such as the Bill \& Melinda Gates Foundation, could remedy the market failure threatening vaccine development for LMICs. Gavi provides one form of subsidy (see 'Spreading the cost'). Support to develop vaccines or to make them available during epidemics is also provided by public organizations, such as the Coalition for Epidemic Preparedness Innovations in Oslo and the Biomedical Advanced Research and Development Authority, part of the US Department of Health and Human Services.

Such schemes need to be expanded and rethought to give vaccine developers more certainty and upfront financial backing. For instance, Gavi could commit to purchasing a vaccine before it has been developed, on the

\section{SPREADING THE COST}

\section{How to fund vaccine development}

The private sector might be able to advance some vaccine candidates for diseases that affect both emerging economies and wealthy nations.

Drug firms have provided many vaccines at a reduced cost to low- and middleincome countries (LMICs) - such as for poliovirus, hepatitis $B$ and pneumococcus - after recovering their research and development investments through sales in wealthy nations. For instance, in the United States alone, the net economic benefit of the oral polio vaccine, licensed in 1961, was estimated to be more than US $\$ 180$ billion by 2006 (ref. 11).

Gaps in resourcing can limit vaccine uptake in LMICs, even for diseases that affect high-income countries. The Haemophilus influenzae type b vaccine was adopted in Gambia, Kenya, Cuba and Nicaragua 12-16 years after it was incorporated into national US immunization programmes. This was largely because of the absence of a global agency able to purchase vaccines for the countries that couldn't afford them, and because suppliers were reluctant to invest in the manufacturing capacity needed for uncertain returns.

Various schemes have now been established, such as Gavi, the Vaccine Alliance, to help make existing vaccines available to people in LMICs. D.C.K. et al. condition that the developers meet certain regulatory milestones. At present, the alliance buys vaccines to distribute to LMICs after they have been licensed or recommended by the WHO for general use.

Regulation is another hurdle. Stakeholders should define clear pathways. They should negotiate more alignment between the various organizations involved in planning, development and oversight. Finally, they should identify what infrastructure and human capacity are needed to ensure that a reliable supply of vaccines can be provided long-term to the people who need them.

Only with this kind of leadership will the global community secure vaccines for some of the world's most debilitating diseases.

David C. Kaslow is vice-president for essential medicines at PATH, Seattle, Washington, USA. Steve Black, David E. Bloom, Mahima Datla, David Salisbury, Rino Rappuoli. e-mail:dkaslow@path.org

1. Nussenzweig, R. S., Vanderberg, J., Most, H. \& Orton, C. Nature 216, 160-162 (1967)

2. Ellis, J. et al. Nature 302, 536-538 (1983).

3. Cochrane, A. H., Santoro, F., Nussenzweig, V., Gwadz, R. W. \& Nussenzweig, R. S. Proc. Natl Acad. Sci. USA 79, 5651-5655 (1982).

4. Stoute, J. A. et al. N. Engl. J. Med. 336, 86-91 (1997).

5. Alonso, P. L. et al. Lancet 364, 1411-1420 (2004).

6. RTS,S Clinical Trials Partnership. Lancet $\mathbf{3 8 6}$, 31-45 (2015).

7. Bloom, D. E., Fan, V. Y. \& Sevilla, J. P. Sci. Transl. Med. 10, eaaj2345 (2018).

8. Trotter, C. L. et al. Lancet Infect. Dis. 17, 867-872 (2017).

9. Mustapha, M. M. \& Harrison, L. H. Hum. Vaccin. Immunother. 14, 1107-1115 (2018).

10. Roberts, L. Science 320, 1710-1715 (2008).

11.Thompson, K. M. et al. Risk Anal. 26, 1571-1580 (2006).

S.B., D.E.B, M.D., D.S. \& R.R. declare competing financial interests; see go.nature.com/2uhcjkw. 